\title{
An expanded-access clinical study of thiotepa (DSP-1958) high-dose chemotherapy before autologous hematopoietic stem cell transplantation in patients with malignant lymphoma
}

\author{
Momoko Nishikori ${ }^{1}$ [ $\cdot$ Yasufumi Masaki ${ }^{2} \cdot$ Nobuharu Fujii $^{3} \cdot$ Takashi Ikeda $^{4} \cdot$ Mariko Takahara-Matsubara $^{5}$. \\ Saori Sugimoto ${ }^{5}$ Eisei Kondo $0^{6,7}$
}

Received: 16 July 2021 / Revised: 14 November 2021 / Accepted: 14 November 2021 / Published online: 26 November 2021

(c) Japanese Society of Hematology 2021

\begin{abstract}
Thiotepa, an antineoplastic ethylenimine alkylating agent that can penetrate the central nervous system, was recently approved in Japan as high-dose chemotherapy prior to autologous hematopoietic stem cell transplantation (HSCT) for patients with malignant lymphoma. To further evaluate the safety and efficacy of thiotepa, a multicenter, open-label, non-comparative, expanded access program was undertaken in Japan, including a larger population of Asian patients with malignant lymphoma. Intravenous thiotepa ( $200 \mathrm{mg} / \mathrm{m}^{2} /$ day) was administered over $2 \mathrm{~h}$ on days -4 and -3 before scheduled HSCT, plus intravenous busulfan $(0.8 \mathrm{mg} / \mathrm{kg})$ over $2 \mathrm{~h}$ every $6 \mathrm{~h}$ on days $-8,-7,-6$ and -5 . In the safety analysis population $(N=51), 25$ patients $(49.0 \%)$ had primary central nervous system lymphomas. The most common treatment-emergent adverse event was febrile neutropenia (49/51 [96.1\%]). No unexpected safety events were observed, and no event resulted in death or treatment modification. Forty-seven patients (92.2\%) had engraftment (neutrophil count $\geq 500 / \mathrm{mm}^{3}$ for three consecutive days after bone-marrow suppression and HSCT). The survival rate at day 100 post-transplantation was $100 \%$. These data confirm the safety of thiotepa prior to autologous HSCT for patients with malignant lymphoma.
\end{abstract}

Trial registration: JapicCTI-173654.

Keywords Hematopoietic stem cell transplantation $\cdot$ Lymphoma $\cdot$ Thiotepa $\cdot$ Transplantation $\cdot$ Autologous

Momoko Nishikori

nishikor@kuhp.kyoto-u.ac.jp

1 Department of Hematology and Oncology, Graduate School of Medicine, Kyoto University, Kyoto 606-8507, Japan

2 Department of Hematology and Immunology, Kanazawa Medical University, Kanazawa, Japan

3 Division of Blood Transfusion, Okayama University Hospital, Okayama, Japan

4 Division of Hematology and Stem Cell Transplantation, Shizuoka Cancer Center, Shizuoka, Japan

5 Sumitomo Dainippon Pharma Co., Ltd., Osaka, Japan

6 Department of Hematology, Kawasaki Medical School, Kurashiki, Japan

7 Department of General Medicine, Graduate School of Medicine, Dentistry and Pharmaceutical Sciences, Okayama University, Okayama, Japan

\section{Introduction}

For patients with high-risk lymphoma, high-dose-chemotherapy (HDT) and autologous hematopoietic stem cell transplantation (HSCT) are administered with curative intent or with the aim of improving survival duration [1-3].

Thiotepa (DSP-1958), an antineoplastic ethylenimine alkylating agent that inhibits DNA synthesis [4], is used as HDT before autologous HSCT in patients with solid or hematologic malignancies [5]. It has the capacity to cross the blood-brain barrier, and is able to rapidly penetrate into the central nervous system (CNS) [6, 7]. Thiotepa has a lengthy medical history, having first been used for the treatment of leukemia [8] and solid tumors [9] in the 1950s. In Japan, thiotepa has been approved for standard-dose chemotherapy since 1958, but manufacturing had been discontinued in $2008[10,11]$. However, thiotepa was approved in Europe in 2010 as HDT prior to HSCT in adult and pediatric patients [12]. As a result, oncologists in Japan felt that there was a 
high medical need for thiotepa as HDT prior to autologous HSCT, especially for the treatment of CNS lymphoma.

We have previously reported data from a phase 1 study of a thiotepa-containing HDT regimen in nine patients with pediatric solid tumors and 10 adult patients ( $\geq 16$ years) with malignant lymphoma [5]. The results indicated that thiotepa was well-tolerated; the bone-marrow suppression rate was $100 \%$ for both pediatric solid tumors and for adult malignant lymphoma, and the engraftment rates were $66.7 \%$ and $100 \%$, respectively. Furthermore, the survival rate at day 100 postHSCT was $77.8 \%$ for pediatric solid tumors and $100 \%$ for malignant lymphoma. Thiotepa was subsequently approved in Japan as HDT prior to autologous HSCT for pediatric malignant solid tumors in March 2019 and for malignant lymphoma in March 2020 [13].

An expanded access program was initiated to further evaluate the safety and efficacy of thiotepa HDT in larger patient populations prior to gaining marketing approval. The study included pediatric patients with solid tumors or brain tumors and adult patients ( $\geq 16$ years) with malignant lymphoma; herein, we report data from the patients with malignant lymphoma.

\section{Patients and methods}

\section{Patients}

The key inclusion criteria were male or female patients aged $\geq 16$ years with malignant lymphoma who had completed hematopoietic cell collection for autologous HSCT; Eastern Cooperative Oncology Group (ECOG) performance status (PS) of 0-2 assessed within 14 days before enrollment; and normal hepatic, renal, and cardiac function (aspartate aminotransferase and alanine aminotransferase $\leq 3$-fold, $\gamma$-glutamyl transferase $\leq 2.5$-fold, and total bilirubin and creatinine $\leq 1.5$-fold the reference range at the clinical site; left ventricular ejection fraction $[\mathrm{LVEF}] \geq 50 \%$ ) based on tests performed within 14 days before enrollment. All patients provided written informed consent prior to study enrollment; for patients aged 16-19 years, written informed consent was also required from their legal representative.

Key exclusion criteria were patients who had undergone any treatment (other than hematopoietic cell collection) for the underlying disease within 13 days before the start of study treatment; previous HSCT within the 6 months prior to the study; receipt of live attenuated vaccine within 90 days or any investigational agent within 27 days before the start of study treatment; pregnancy or lactation; present or previous history of complications affecting drug metabolism or excretion; active infection; presence of human immunodeficiency virus antibody, hepatitis B surface antigen or antibody, or hepatitis B core antibody (although patients positive for hepatitis B surface antigen or antibody, or hepatitis B core antibody could be enrolled if vaccinated for type B hepatitis); pericardial effusion, pleural effusion, or ascites requiring treatment or receipt of treatment for these conditions within 14 days before enrollment; hypersensitivity to thiotepa, macrogol 400, or busulfan; and any other reason which may endanger the patient or confound the study outcomes based on the judgement of the study investigator.

\section{Study design and treatments}

This was a multicenter, open-label, non-comparative, expanded access program (JapicCTI-173654) which was conducted in patients with malignant lymphoma at four institutions in Japan between September 2017 and June 2020. The study protocol and related documentation were approved by the ethics committee or independent review board of each study center. The study was conducted in accordance with the Declaration of Helsinki, Good Clinical Practice guidelines, and all applicable legal and regulatory requirements.

The study design is shown in Fig. 1A. The study comprised a HDT period and a transplant period, with the day of HSCT defined as day 0 . Thiotepa $\left(200 \mathrm{mg} / \mathrm{m}^{2} /\right.$ day $)$ was administered intravenously (IV) over $2 \mathrm{~h}$ on day -4 and day -3 before scheduled HSCT. Busulfan $(0.8 \mathrm{mg} / \mathrm{kg})$ was administered IV over $2 \mathrm{~h}$ every $6 \mathrm{~h}$ (i.e., four times daily, for a total dose of $3.2 \mathrm{mg} / \mathrm{kg} / \mathrm{day}$ ) on days $-8,-7,-6$ and -5 before the scheduled HSCT. The doses of thiotepa and busulfan could be reduced or interrupted if deemed necessary; such decisions were made at the investigator's discretion and no specific dosage modification instructions were mandated by the protocol.

Prophylactic administration of anticonvulsants (e.g., sodium valproate) was recommended in the period between $48 \mathrm{~h}$ before and $48 \mathrm{~h}$ after administration of busulfan, as this drug may cause seizures in patients with malignant lymphoma [14].

\section{Endpoints}

The primary study objective was to assess the safety of IV thiotepa as HDT before autologous HSCT in patients with malignant lymphoma. Safety was assessed by recording treatment-emergent adverse events (TEAEs) and adverse drug reactions (ADRs) occurring between the start of study treatment and day 28 post-HSCT, evaluating ECOG PS, and assessing the results of physical and laboratory tests (12-lead electrocardiogram [ECG], LVEF, laboratory measures, vital signs, and body weight). TEAEs were classified using the Medical Dictionary for Regulatory Activities (MedDRA) version 19.1. In this study, the myelosuppression rate was set as an efficacy endpoint and, therefore, hematologic toxicity 
A
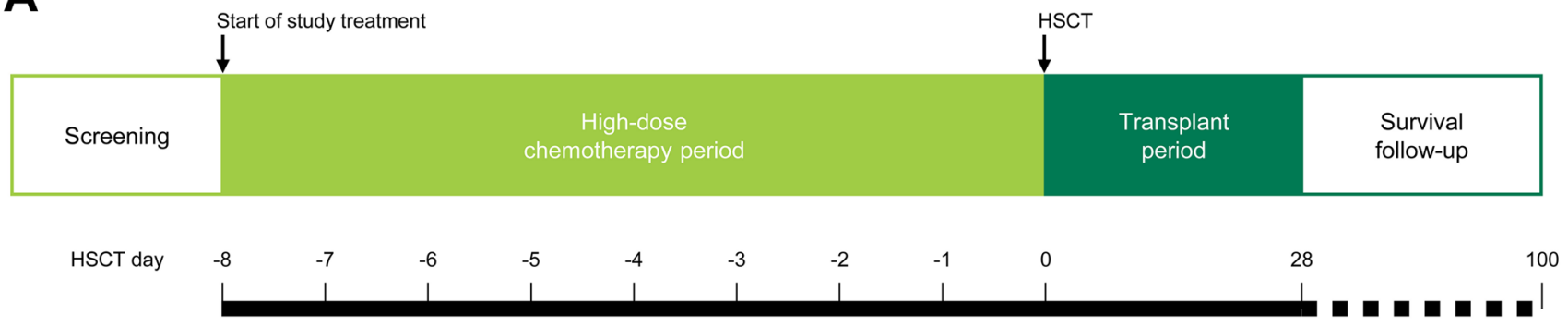

Thiotepa ${ }^{a}$

Busulfan ${ }^{b}$

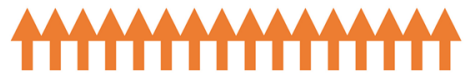

B

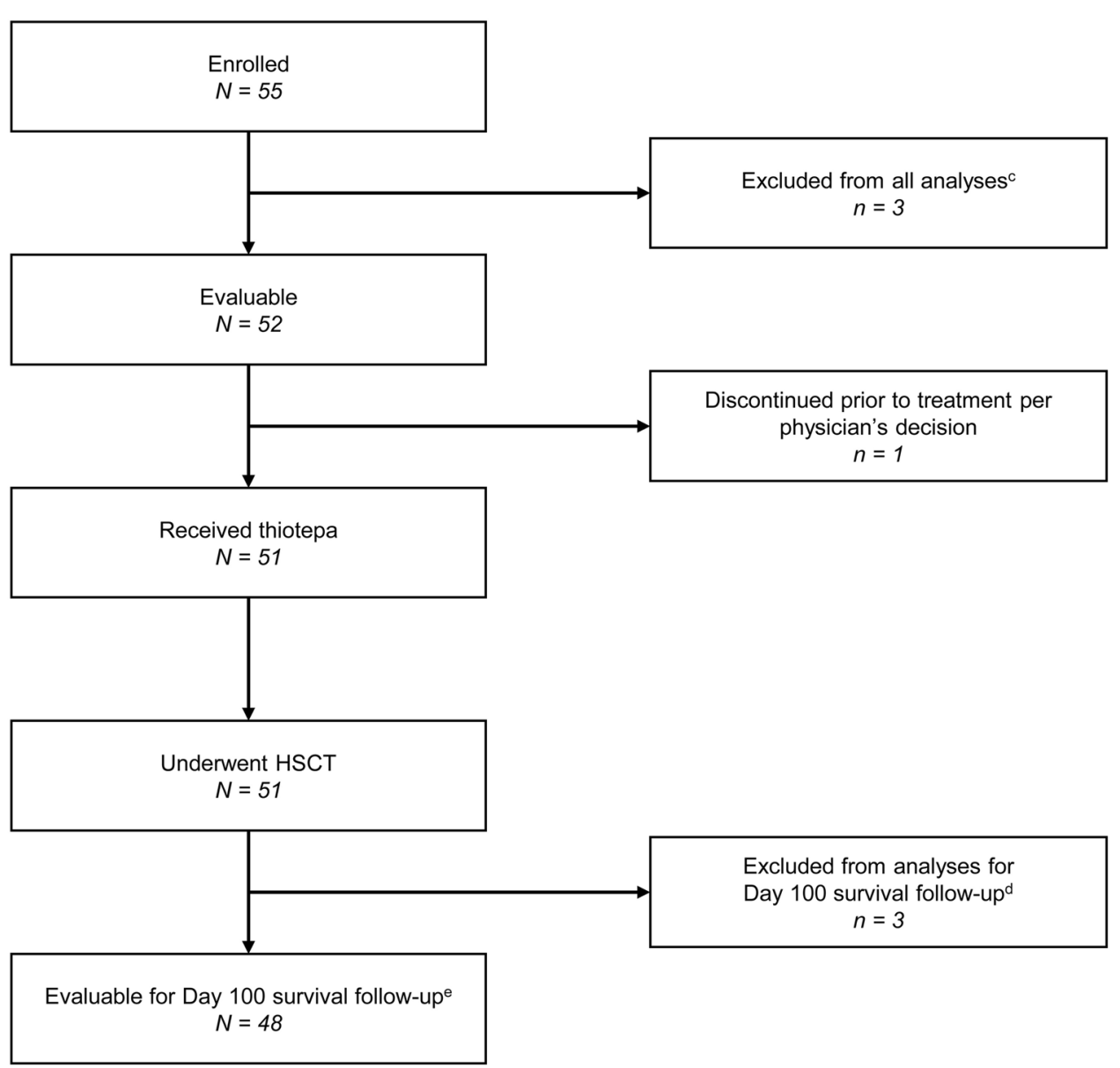

Fig. 1 A Study design in patients with malignant lymphoma. B Patient disposition. The screening period included collection of informed consent and study enrollment. Day 0 was the day of HSCT.

${ }^{a}$ Thiotepa $200 \mathrm{mg} / \mathrm{m}^{2} /$ day IV over $2 \mathrm{~h}$ on days -4 and -3 . ${ }^{\mathrm{b}}$ Busulfan $0.8 \mathrm{mg} / \mathrm{kg}$ IV over $2 \mathrm{~h}$ every $6 \mathrm{~h}$ on days $-8,-7,-6$ and -5 . ${ }^{\mathrm{c}}$ Three patents did not have validated data; the sponsor was unable to visit one of the study sites to verify the documentation for most of the data due to the institute's COVID-19 precautions within the study period. Therefore, these patients were excluded from all analyses. ${ }^{\mathrm{d}}$ Three additional patients did not have validated data for survival at day 100 after HSCT due to the COVID-19 precautions at the same institute. These patients were excluded from the analysis of survival at day 100 after HSCT. Validated data for other measures, including adverse events, were available and these three patients were included in the safety analysis set. ${ }^{e}$ Includes two patients who did not complete the full 100 days (censored) due to termination of the study when thiotepa received regulatory approval for malignant lymphoma. HDT high-dose chemotherapy, HSCT hematopoietic stem cell transplantation, $I V$ intravenously 
associated with thiotepa-based HDT prior to HSCT was not included in the evaluation of TEAEs.

The secondary objective was to assess the efficacy of IV thiotepa in this patient population. Efficacy endpoints included the bone-marrow suppression rate (defined as the proportion of patients with a neutrophil count $<500 / \mathrm{mm}^{3}$ at least once during the 28 days after HSCT), the engraftment rate (defined as the proportion of patients with a neutrophil count $\geq 500 / \mathrm{mm}^{3}$ for 3 consecutive days after bone-marrow suppression and HSCT), time to engraftment (evaluated in those patients with evidence of engraftment, and defined as the number of days between HSCT and the first of three consecutive days with a neutrophil count $\geq 500 / \mathrm{mm}^{3}$ after bone-marrow suppression and HSCT), and the survival rate at day 100 post-HSCT.

\section{Statistical methods}

The overall sample size of approximately 100 patients (including patients with pediatric solid tumors and those with malignant lymphoma) was determined based on the expected number of participants; no formal study size calculations or hypothesis testing was performed.

The safety analysis population included all patients who received at least a single dose of thiotepa. The number and frequency of AEs and ADRs were summarized; physical and laboratory test results were reported as summary statistics and ECG interpretations as shift tables.

For the efficacy analyses of bone-marrow suppression rate, engraftment rate and time to engraftment, the safety analysis population was used, and missing data were not imputed. The rate of survival at day 100 post-HSCT was estimated using Kaplan-Meier methodology. Death after HSCT (regardless of cause of death) was defined as an event, and observations were censored on the latest date of confirmed survival. Statistical analyses were conducted using SAS software version 9.4 (SAS Institute Inc., Cary. NC, USA).

\section{Results}

\section{Patients}

A total of 55 patients with malignant lymphoma were enrolled into the study. Patient disposition is shown in Fig. 1B. One patient discontinued due to physician decision prior to receiving thiotepa. Three patients from one study site were excluded from all analyses, because the sponsor was unable to verify the documentation due to the institute's COVID-19 precautions within the study period. For the same reason, three additional patients at the same institute did not have validated data for survival at day 100 after
HSCT, and were excluded from the analysis of survival at day 100 after HSCT. However, as these three patients had validated data for other measures including TEAEs, it was considered that it was ethically and medically desirable to include them in the safety analysis set. Thus, the safety analysis population comprised 51 patients, all of whom underwent HSCT, and the survival analysis population comprised 48 patients. It should also be noted that the survival analysis at day 100 post-HSCT included two patients who did not complete the full 100 days (censored) due to termination of the study when thiotepa received regulatory approval for malignant lymphoma.

Baseline demographics and clinical characteristics for the safety analysis set are shown in Table 1. Overall, 34/51 (66.7\%) patients were male, the median age was 54 years (range 38-72), and 30/51 (58.8\%) patients had an ECOG PS

Table 1 Baseline demographics and clinical characteristics (safety analysis set)

\begin{tabular}{lc}
\hline & $\begin{array}{c}\text { Patients with malig- } \\
\text { nant lymphoma } \\
(N=51)\end{array}$ \\
\hline Sex (male) & $34(66.7)$ \\
Age (years), median (min, max) & $54.0(38,72)$ \\
$\geq 65$ years, $n(\%)$ & $15(29.4)$ \\
Height $(\mathrm{cm})$, median (min, max) & $167.1(150.9,185.1)$ \\
Weight $(\mathrm{kg})$, median $(\min , \max )$ & $62.9(36.3,95.1)$ \\
BSA (m $\left.{ }^{2}\right)^{\mathrm{a}}$, median $(\min , \max )$ & $1.7(1.3,2.1)$ \\
ECOG PS & \\
0 & $30(58.8)$ \\
1 & $18(35.3)$ \\
2 & $3(5.9)$ \\
Disease type & \\
New onset & $27(52.9)$ \\
Relapse & $24(47.1)$ \\
Prior HSCT transplantations & \\
0 & $48(94.1)$ \\
1 & $3(5.9)$ \\
Complications & \\
Constipation & $48(94.1)$ \\
Hypertension & $24(47.1)$ \\
Insomnia & $11(21.6)$ \\
Neuropathy peripheral & $10(19.6)$ \\
Hyperlipidemia & $10(19.6)$ \\
\hline & $6(11.8)$ \\
\hline
\end{tabular}

All patients were Asian. Number of patients (\%) are shown unless otherwise indicated

BSA body surface area, ECOG PS Eastern Cooperative Oncology Group performance status, HSCT hematopoietic stem cell transplantation

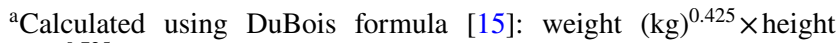
$(\mathrm{cm})^{0.725} \times 0.007184$

${ }^{\mathrm{b}}$ Complications occurring in $\geq 10 \%$ of patients are shown 
of 0 . HSCT was reported as a treatment for relapsed disease in $24 / 51(47.1 \%)$ patients, and $3 / 51(5.9 \%)$ had received one prior HSCT transplantation. The type of lymphoma is shown in Supplementary Table 1. Overall, 25/51 patients (49.0\%) had primary CNS lymphoma (PCNSL), and several of the remaining 26 patients also had CNS lesions.

\section{Treatments}

The dosing schedules for thiotepa and busulfan are shown in Table 2 . All patients in the safety analysis set received 2 days of thiotepa and 4 days of busulfan treatment; median dose intensities for both were $100 \%$.

Dose reductions were uncommon. For thiotepa, 4/51 patients had a reduction from $200 \mathrm{mg} / \mathrm{m}^{2} /$ day, with three of these patients receiving $160 \mathrm{mg} / \mathrm{m}^{2} /$ day $(80 \%$ of the expected dose) and one patient receiving $140 \mathrm{mg} / \mathrm{m}^{2} /$ day $(70 \%$ of the expected dose). For busulfan, 7/51 patients had a dose reduction from $3.2 \mathrm{mg} / \mathrm{kg} / \mathrm{day}$; of these, five patients received $2.56 \mathrm{mg} / \mathrm{kg} /$ day $(80 \%$ of the expected dose), and two patients received $2.24 \mathrm{mg} / \mathrm{kg} /$ day (70\% of the expected dose).

Concomitant granulocyte colony stimulating factor (filgrastim or lenograstim) was received by $45 / 51$ (93.8\%) patients in this analysis.

\section{Safety}

TEAEs occurring in $\geq 10 \%$ of patients are shown in Table 3 . The most frequently reported TEAE was febrile neutropenia (49/51 [96.1\%] patients); all patients were recorded as having had grade 3 events. Although diarrhea (42/51 [82.4\%]), stomatitis (32/51 [62.7\%]), nausea (29/51 [56.9\%]), and decreased appetite (28/51 [54.9\%]) all occurred in more than half of patients, many of these events were grade 1 or 2 .

No TEAEs resulting in death or treatment discontinuation or interruption, or dose reduction, were reported during the study. Four patients reported serious TEAEs up to day 28 post-HSCT (hepatic veno-occlusive disease: grade $3, n=1$, grade $4, n=1$; hepatic dysfunction: grade $4, n=1$; hypoxia and viral infection: grade 3 and grade 4 , respectively, $n=1$ ). The cases of veno-occlusive disease were considered
Table 3 Summary of TEAEs (safety analysis set)

\begin{tabular}{lll}
\hline MedDRA preferred term & \multicolumn{2}{l}{$\begin{array}{l}\text { Patients with malignant } \\
\text { lymphoma }(N=51)\end{array}$} \\
\cline { 2 - 3 } & All grades & Grade 3 or 4 \\
\hline Any TEAE & $51(100.0)$ & $51(100.0)$ \\
TEAEs occurring in $\geq 10 \%$ of patients & & \\
Febrile neutropenia & $49(96.1)$ & $49(96.1)$ \\
Diarrhea & $42(82.4)$ & $6(11.8)$ \\
Stomatitis & $32(62.7)$ & $13(25.5)$ \\
Nausea & $29(56.9))$ & $4(7.8)$ \\
Decreased appetite & $28(54.9)$ & $16(31.4)$ \\
Malaise & $24(47.1)$ & 0 \\
Dysgeusia & $19(37.3)$ & 0 \\
Hepatic function abnormal & $17(33.3)$ & $5(9.8)$ \\
Vomiting & $15(29.4)$ & $1(2.0)$ \\
Hypokalemia & $12(23.5)$ & $3(5.9)$ \\
Oropharyngeal pain & $12(23.5)$ & $3(5.9)$ \\
Pharyngitis & $12(23.5)$ & $3(5.9)$ \\
Pyrexia & $11(21.6)$ & 0 \\
Hypersensitivity & $10(19.6)$ & $1(2.0)$ \\
Dermatitis contact & $9(17.6)$ & 0 \\
Insomnia & $9(17.6)$ & 0 \\
Proctalgia & $9(17.6)$ & 0 \\
Rash & $7(13.7)$ & 0 \\
Headache & $6(11.8)$ & 0 \\
\hline
\end{tabular}

No grade 5 events were reported. Number of patients (\%) are shown MedDRA Medical Dictionary for Regulatory Activities, TEAE treatment-emergent adverse event

possibly related to study treatment (ADRs), most likely due to busulfan administration, while the hepatic dysfunction was considered unlikely to be related to study treatment. None of these events had resolved/recovered at the end of the study (i.e., day 28 post-HSCT), but all resolved/recovered during the survival follow-up period. The viral infection was caused by human herpesvirus 6; it was considered possibly related to treatment (ADR), since herpesvirus reactivation in immunodeficient patients is known to be possible. The infection was subsequently reported to be recovered; the event
Table 2 Treatment details (safety analysis set)

\begin{tabular}{lll}
\hline & Thiotepa & Busulfan \\
\hline Number of exposure days, $n(\%)$ & & \\
1 & 0 & 0 \\
2 & $51(100.0)$ & 0 \\
3 & - & 0 \\
4 & - & $51(100.0)$ \\
Daily dose $\left(\mathrm{mg} / \mathrm{m}^{2} /\right.$ day $)$, median $(\min , \max )$ & $200(140,200)$ & $3.2(2.2,3.2)$ \\
Cumulative dose $\left(\mathrm{mg} / \mathrm{m}^{2}\right)$, median $(\min , \max )$ & $400(280,400)$ & $12.8(9.0,12.8)$ \\
Dose intensity $(\%)$, median $(\min , \max )$ & $100(70,100)$ & $100(70,100)$ \\
\hline
\end{tabular}


of hypoxia was considered unrelated to treatment and had resolved by the end of the study.

One patient had an abnormal, clinically significant ECG change on day 7 post-HSCT, but no longer had a clinically significant abnormality by day 28 . No other clinically significant changes in physical or laboratory test values were noted during the study.

\section{Efficacy}

Efficacy outcomes are summarized in Table 4. All 51 patients $(100.0 \%)$ achieved bone-marrow suppression and $47 / 51(92.2 \%)$ had engraftment. The median time to engraftment in those 47 patients was 11.0 days. The remaining four patients did not meet the narrow definition of engraftment used in this study (a neutrophil count $\geq 500 / \mathrm{mm}^{3}$ for 3 consecutive days), because blood sampling was not performed on 3 consecutive days; thus the corresponding consecutive neutrophil counts were unavailable. However, each of the four did achieve a neutrophil count $\geq 500 / \mathrm{mm}^{3}$ at all ( $>3$ ) time points evaluated after autologous HSCT day 11. The median follow-up after autologous HSCT was 109 days. The survival rate at day 100 post-HSCT was $100 \%$ (Supplementary Fig. 1).

\section{Discussion}

In this expanded access study, the safety of IV thiotepa as HDT before autologous HSCT in patients with malignant lymphoma was assessed. This was the largest study to date undertaken in Japan of Asian patients treated with a thiotepa and busulfan HDT regimen, and the resulting data provide important information for oncologists when choosing the optimal treatment for their patients with malignant lymphoma.

The safety profile of this treatment regimen was acceptable. The most frequently reported TEAEs (febrile neutropenia and gastrointestinal toxicities) were consistent with those reported in adults with malignant lymphoma in the phase I study [5], and with the European [12] and Japanese [13] product labels. No unexpected safety outcomes were observed. Two patients in this study had an event of hepatic veno-occlusive disease; this event is a known risk in some patients during HDT or following HSCT [16]. No patients discontinued treatment or died due to an AE in this study.

A previous single-center analysis of data from 24 patients treated with thioteopa plus busulfan high-dose chemotherapy, which included patients drawn from the phase 1 pharmacokinetic study [5] and also from this expanded access program, reported the occurrence of secondary failure of platelet recovery (SFPR) in three of the patients evaluated after the end of the study period [17]. SFPR is known to be a complication of hematopoietic stem cell transplantation, although it generally occurs more frequently in allogenic than in autologous transplants [18]. As it was considered possible that the development of SFPR may have been associated with the use of busulfan [17], clinicians using a thiotepa plus busulfan high-dose chemotherapy regimen prior to autologous HSCT should be aware of the possibility of SFPR and ensure that timely treatment can be initiated.

The efficacy results indicated that using thiotepa in this regimen was able to contribute to good clinical outcomes for patients, with all patients achieving bone-marrow suppression and $>90 \%$ having engraftment. Furthermore, the survival rate at day 100 post-HSCT was also $100 \%$. Although a total of six patients were excluded from the formal analysis of the survival rate at day 100 post-HSCT due to a lack of validated data from one site (resulting from the institute's COVID-19 precautions), all six remained alive at this time point. These findings confirm the efficacy data observed in the prior phase 1 study [5].

Due to its potent ability to penetrate the CNS $[6,7]$, thiotepa shows promise for the treatment of PCNSL, and other lymphomas in which lesions are found within the nervous system. Although PCNSL is relatively rare [19], other types of malignant lymphoma can recur within the CNS [20-22], and survival times are often poor [19-21]. Thus, a treatment which can provide benefit to these patients would be a valuable addition to the current therapeutic algorithms. In
Table 4 Summary of efficacy outcomes (safety analysis set)

\begin{tabular}{ll}
\hline & $\begin{array}{l}\text { Patients with malignant lymphoma } \\
(N=51)\end{array}$ \\
\hline Bone-marrow suppression, $n(\%)[95 \% \mathrm{CI}]$ & $51(100.0)[93.0,100.0]$ \\
Neutrophil engraftment ${ }^{\mathrm{a}}, n(\%)[95 \% \mathrm{CI}]$ & $47(92.2)[81.1,97.8]$ \\
Time to engraftment (days), median (min, max) & $11.0(8,17)$ \\
Evaluable subjects at day 100 post-HSCT, $n$ & 48 \\
Survival at day $100, n(\%)[95 \% \mathrm{CI}]$ & $48(100.0)[-,-]$ \\
\hline
\end{tabular}

${ }^{a}$ Defined as the proportion of patients with a neutrophil count $\geq 500 / \mathrm{mm}^{3}$ for 3 consecutive days after bonemarrow suppression and HSCT

CI confidence interval, HSCT hematopoietic stem cell transplantation 
this study, $49.0 \%$ of patients had PCNSL and several others also had CNS lesions; given that 100-day survival in evaluable patients was $100 \%$, these data suggest that thiotepa may be beneficial for the treatment of patients with CNS-based disease.

The main study limitations include that the study was conducted in Japan and enrolled only Asian patients with malignant lymphoma, which means that the data may not be generalizable to the global patient population. The 100-day post-HSCT follow-up means that long-term survival data are not available, and further studies are warranted to evaluate outcomes over longer durations. It is possible that the implementation of emergency restrictions due to COVID-19, and patient censoring at study termination (due to drug approval by the regulatory authorities), may have confounded the outcome calculations, but since a very small number of patients were affected, we consider that these events had a limited impact on the overall study findings.

In conclusion, the data from this expanded access study confirmed the safety of IV thiotepa as part of the HDT regimen prior to autologous HSCT for patients with malignant lymphoma in Japan. The results confirmed the data reported in the phase I study, but in a larger population, including many patients with CNS-based disease. There were no new safety concerns, and we consider that thiotepa is suitable for administration to this patient population.

Supplementary Information The online version contains supplementary material available at https://doi.org/10.1007/s12185-021-03263-y.

Acknowledgements This study was funded by Sumitomo Dainippon Pharma Co., Ltd. The authors would like to express our sincere gratitude to all patients and their families who participated in this study. We thank Sally-Anne Mitchell, PhD (McCANN HEALTH CMC, Japan) for providing medical writing support, which was funded by Sumitomo Dainippon Pharma Co., Ltd., in compliance with Good Publication Practice 3 guidelines (https://www.ismpp.org/gpp3).

\section{Declarations}

Conflict of interest YM has received research grants from Kyowa Kirin Pharma, Astellas Pharma, Eisai Pharma, Ono Pharma, Pfizer Pharma, Asahi Kasei Pharma, MSD Pharma, Daiichi-Sankyo Pharma, Taisho Pharma, Taiho Pharma, Takeda Pharma, Chugai Pharma, Teijin Pharma, Nippon Kayaku, and Mochida Pharma, outside the submitted work. MT and SS are employees of Sumitomo Dainippon Pharma Co., Ltd. EK received honoraria from Sumitomo Dainippon Pharma and Otsuka. MN, NF, TI, have nothing to declare.

\section{References}

1. Jantunen E, Sureda A. The evolving role of stem cell transplants in lymphomas. Biol Blood Marrow Transplant. 2012;18(5):660-73.
2. Benekou K, Montoto S. Role of stem cell transplant in lymphoma in the era of new drugs. Curr Opin Oncol. 2017;29(6):455-9.

3. Zahid U, Akbar F, Amaraneni A, Husnain M, Chan O, Riaz IB, et al. A review of autologous stem cell transplantation in lymphoma. Curr Hematol Malig Rep. 2017;12(3):217-26.

4. Maanen MJ, Smeets CJ, Beijnen JH. Chemistry, pharmacology and pharmacokinetics of N, N', N" -triethylenethiophosphoramide (ThioTEPA). Cancer Treat Rev. 2000;26(4):257-68.

5. Kondo E, Ikeda T, Goto H, Nishikori M, Maeda N, Matsumoto $\mathrm{K}$, et al. Pharmacokinetics of thiotepa in high-dose regimens for autologous hematopoietic stem cell transplant in Japanese patients with pediatric tumors or adult lymphoma. Cancer Chemother Pharmacol. 2019;84(4):849-60.

6. Heideman RL, Packer RJ, Reaman GH, Allen JC, Lange $\mathrm{B}$, Horowitz ME, et al. A phase II evaluation of thiotepa in pediatric central nervous system malignancies. Cancer. 1993;72(1):271-5.

7. Prodduturi P, Bierman PJ. Current and emerging pharmacotherapies for primary CNS lymphoma. Clin Med Insights Oncol. 2012;6:219-31.

8. Shay H, Zarafonetis C, Smith N, Woldow I, Sun DC. Treatment of leukemia with triethylene thiophosphoramide (thio-TEPA); preliminary results in experimental and clinical leukemia. AMA Arch Intern Med. 1953;92(5):628-45.

9. Shay H, Sun DC. Clinical studies of triethylenethiophosphoramide in the treatment of inoperable cancer. Cancer. 1955;8(3):498-511.

10. Kondo E. Primary central nervous system lymphoma: advances in treatment strategies. Rinsho Ketsueki. 2020;61(5):510-9 (In Japanese).

11. Suzuki R, Takahashi Y, Inoue M, Kanamori H, Hashii Y, Sakamaki H, et al. Thiotepa for autologous hematopoietic stem cell transplantation for solid tumors. Ann Oncol. 2015;26(suppl 7):vii106-51.

12. TEPADINA $15 \mathrm{mg}$ powder for concentrate for solution for infusion (summary of product characteristics). ADIENNE S.r.1. S.U, Caponago, Italy; 2015. https://www.ema.europa.eu/en/docum ents/product-information/tepadina-epar-product-information en.pdf. Accessed 19 June 2021.

13. Thiotepa $100 \mathrm{mg}$ (RETHIO IV infusion) [Japanese package insert]. Sumitomo Dainippon Pharma Co., Ltd., Osaka, Japan; 2020. https://pins.japic.or.jp/pdf/newPINS/00068017.pdf. Accessed 19 June 192021.

14. Ciurea SO, Andersson BS. Busulfan in hematopoietic stem cell transplantation. Biol Blood Marrow Transplant. 2009;15(5):523-36.

15. Du Bois D, Du Bois EF. A formula to estimate the approximate surface area if height and weight be known. Nutrition. 1989;5(5):303-11.

16. Dalle JH, Giralt SA. Hepatic veno-occlusive disease after hematopoietic stem cell transplantation: risk factors and stratification, prophylaxis, and treatment. Biol Blood Marrow Transplant. 2016;22(3):400-9.

17. Wada F, Nishikori M, Hishizawa M, Watanabe M, Aiba A, Kitano T, et al. Secondary failure of platelet recovery in patients treated with high-dose thiotepa and busulfan followed by autologous stem cell transplantation. Int J Hematol. 2020;112(5):609-13.

18. Bernstein SH, Nademanee AP, Vose JM, Tricot G, Fay JW, Negrin RS, et al. A multicenter study of platelet recovery and utilization in patients after myeloablative therapy and hematopoietic stem cell transplantation. Blood. 1998;91(9):3509-17.

19. Choi YS. Recent advances in the management of primary central nervous system lymphoma. Blood Res. 2020;55(S1):S58-62. 
20. Kim SJ, Oh SY, Kim JS, Kim H, Lee GW, Won JH, et al. Secondary central nervous system (CNS) involvement in patients with diffuse large B-cell lymphoma: a therapeutic dilemma. Ann Hematol. 2011;90(5):539-46.

21. Ferreri AJM. Secondary CNS lymphoma: the poisoned needle in the haystack. Ann Oncol. 2017;28(10):2335-7.

22. van Blydenstein SA, Patel M, Philip V, Lakha A, Pather S, Westgarth-Taylor T, et al. Classical Hodgkin lymphoma involving the central nervous system (brain) —an unusual presentation. Clin Case Rep. 2014;2(3):88-92.

Publisher's Note Springer Nature remains neutral with regard to jurisdictional claims in published maps and institutional affiliations. 